\title{
MODELING AND ON-LINE MONITORING OF MACHINED SURFACE IN TURNING OPERATIONS
}

\author{
Avisekh Banerjee \\ University of Western Ontario \\ London, Ontario, Canada \\ abanerj@uwo.ca \\ Evgueni V. Bordatchev \\ National Research Council of Canada, \\ Integrated Manufacturing Technologies Institute \\ London, Ontario, Canada \\ evgueni.bordatchev@nrc.gc.ca \\ Sounak Kumar Choudhury \\ Indian Institute of Technology, Kanpur, India \\ choudhry@itk.ac.in
}

\begin{abstract}
Machined suface profile and roughness are important parameters in evaluating the quality of a machining operation. They are resulted from the transformation of the complex tool-workpiece displacements involving the dynamics of the machine tool mechanical system, cutting process, and cutting motions. The focus of this study is the fundamental understanding of the surface profile formation during turning and development of regression and neural network (NN) models of strface roughness incorporating the effects of cutting parameters and tool-workpiece displacements. Also, a bifurcated optoelectrical transdicer was developed for on-line monitoring of surface roughness based on the scattering of laser beams from machined surface. The feasibility of on-line monitoring was studied by comparing with actual roughness as well as the prediction restits of the regression and $N N$ models.
\end{abstract}

\section{INTRODUCTION}

The geometric quality of the machined part with respect to its accuracy, precision and surface finish, is one of the most important measures of the machine tool performance, functionality and final acceptability of the product. Therefore, advanced CNC-based machining technologies will require reliable sensor and control technologies to monitor and control cutting process parameters, and thereby measure and predict the actual surface finish. To achieve un-manned machining, surface roughness, needs to be monitored on-line during the cutting process, so that in case of deviation from the desired limits, control action could be taken.

The actual surface roughness profile can be measured by on-line monitoring the

Please use the following format when citing this chapter:

Banerjee, A., Bordatchev, E. V., Choudhury, S. K., 2006, in IFIP International Federation for Information Processing, Volume 220, Information Technology for Balanced Manufacturing Systems, ed. Shen, W., (Boston: Springer), pp. 489-498. 
relative distance of the surface irregularities from a reference plane parallel to the surface with the help of laser beam (Shriashi, 1981, Lee, 1987). An indirect on-line roughness measuring technique based on cutting vibration (Jang, 1996) has also been proposed, as a convenient alternative to direct measurement.

Due to the technical difficulties in on-line monitoring of surface roughness, mathematical modeling of surface roughness based on cutting parameters, for example, first and second order surface regression models (Choudhury, 1997), has been proposed for turning operations. The relative radial displacement between tool and the workpiece (Sata, 1985), cutting vibration (Chen, 2001) and machine tool vibration (Abouelatta, 2001) play an important role in the generation of the surface roughness and had been included as an input to the regression models (Lin, 1998). Apart from regression models, evolutionary computational tool, e.g., neural networks (NN) (Rangwala, 1981, Dimla, 1997, Risbood, 2003), has also been proposed owing to the non-linearity of the cutting process, making the prediction of the surface roughness difficult.

In this paper, both the modeling of surface roughness and its on-line measurements with bifurcated opto-electrical transducer are considered for the purpose of on-line monitoring of machined surface in turning operations. The feasibility of the proposed approach is studied by analyzing the predicted error obtained from regression and NN modeling and measurement error with respect to the actual surface roughness.

\section{MODELING}

\subsection{Systematic Approach}

The only task of any machine tool is the production of a workpiece with desired geometric quality. Therefore, modeling of the machining performance should be focused on analysis and prediction of the actual surface roughness. Figure 1 shows a systematic representation of the surface profile formation as a dynamic process involving the dynamics of the machine tool mechanical system and the cutting process. The machine tool mechanical system has three principal components " $\mathrm{A}$ ", "B", and "C" representing the spindle, carriage, and the tailstock respectively,

Based on the systematic representation and making use of the differential operator $p=d / d t$, dynamics of the machining process with respect to surface formation can be expressed by the following system:

$$
\left\{\begin{array}{l}
\mathbf{x}(t)-\mathbf{W}(p) \mathbf{f}(t)=0 \\
\mathbf{f}(t)=\mathbf{f}^{*}(t)-\mathbf{G}(p) \mathbf{x}(t) \\
\mathbf{r}(t)=\mathbf{Q}(p)\left(\mathbf{x}(t)+\mathbf{u}(t)+\mathbf{u}^{*}(t)\right) \\
\mathbf{z}(t)=\Sigma(\mathbf{r}(t))
\end{array}\right.
$$

where $\mathbf{W}(p)$ is the $3 \times 3$ differential matrix operator of the machine-tool mechanical system, which transforms the cutting force vector $\mathrm{f}(t)$ into the vector of workpiecetool relative displacements $\mathbf{x}(t)=\left\{x_{1}(t), x_{2}(t), x_{3}(t)\right\} ; \mathbf{G}(p)$ is the $3 \times 3$ differential matrix operator of the cutting process and transforms the $\mathbf{x}(t)$ vector into additional components of the $\mathbf{f}(t)$ vector; $\mathbf{f}^{*}(t)$ is the force noise vector of the cutting process, 
and represents the non-linear properties of the cutting process; $Q(p)$ is the $3 \times 1$ differential matrix operator, which represents the surface profile formation as a dynamic process; $\mathbf{r}(t)$ is the radius vector of the machined surface profile, and results from the control vector $\mathbf{u}(t)$ and applying $\mathbf{Q}(p)$ to $\mathbf{x}(t) ; \mathbf{z}(t)$ is the product quality vector, its components are ovality, cylindricity, roughness, etc., and is finally formed from the workpiece surface profile, represented by $\mathbf{r}(t)$, and by some statistical operator $\Sigma$.

This systematic representation shows that all mathematical models of the surface profile formation should link together along with the actual workpiece-tool displacements and cutting parameters. The representation is used for further development of regression and $\mathrm{NN}$ models presented below.

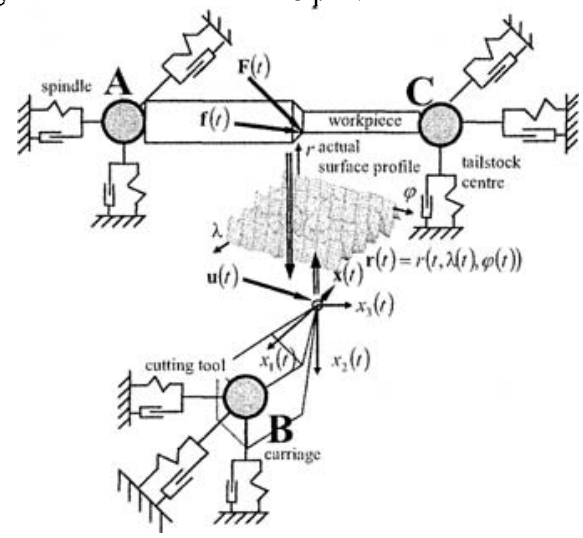

Figure 1 - Formation of surface profile as a dynamic process.

\subsection{Regression Analysis}

Multivariable regression analysis approach is proposed to determine a functional relationship between cutting process parameters (input variables) and surface finish (output parameter), to predict surface roughness. Both log-linear and second order response models have been implemented such that the effect of different independent variables as well as the effect of their interactions with each other on surface roughness can be modeled. The generic models for predicting the surface roughness $R_{a}^{\text {predicted }}=K \cdot v^{\beta_{1}} \cdot f^{\beta_{2}} \cdot \alpha^{\beta_{3}} \cdot \bar{a}_{\text {disp }}^{\beta_{4}}$ (Jang, 1996) based on the measured variables of speed $(v)$, feed $(f)$, rake angle $(\alpha)$, and the averaged acceleration of workpiece-tool displacement $\left(\bar{a}_{\text {disy }}\right)$ can be linearized by taking the logarithm and expressed in terms of a linear regression model as follows:

1) Three regressor model:

a) Multiple log-linear regression model:

$$
\ln \left(R_{a}{ }^{\text {prevelitud }}\right)=\beta_{0}+\beta_{1} \ln (v)+\beta_{2} \ln (f)+\beta_{3} \ln (\alpha)+\varepsilon
$$

b) Second order surface response model:

$$
R_{\mathrm{a}}^{\text {predicted }}=\beta_{0}+\beta_{1} v+\beta_{2} f+\beta_{3} \alpha+\beta_{4} v^{2}+\beta_{5} f^{2}+\beta_{6} \alpha^{2}+\beta_{7} v f+\beta_{8} v \alpha+\beta_{9} f \alpha+\varepsilon
$$

2) Four regressor model: 
a) Multiple log-linear regression model:

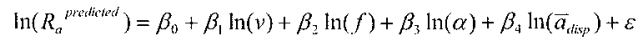

b) Second order surface response model:

$$
\begin{aligned}
R_{\mathrm{a}}^{{ }^{\text {mredicted }}} & =\beta_{0}+\beta_{1} v+\beta_{2} f+\beta_{3} \alpha+\beta_{4} v^{2}+\beta_{5} f^{2}+\beta_{6} \alpha^{2}+\beta_{7} \bar{a}_{d i s p}{ }^{2} \\
& +\beta_{8} v f+\beta_{9} v \alpha+\beta_{10} f \alpha+\beta_{11} v \bar{a}_{d i s p}+\beta_{12} f \bar{a}_{d i s p}+\beta_{13} \alpha \bar{a}_{d i s p}+\varepsilon
\end{aligned}
$$

Regression coefficients $\left(\beta_{i}\right)$ in above models were identified from experimentally obtained data using statistical package MINITA $^{\mathrm{TM}}$.

\subsection{Neural Network}

Neural Network (NN) consists of massive interconnections of simple processing units or neurons. Each connection is associated with a weight, which is adjusted based on previous input-output mapping, thus loosely simulating a human brain. The network can incorporate past experience and subsequently be used to map the inputoutput of any generic problem. The inherent non-linearity of the cutting process even for turning has made $\mathrm{NN}$ well accepted for predicting surface roughness (Dimla, 1997, Risbood, 2003). An output response is generated from a simple processing unit or perceptrons when the sum of the weighted input signals $\left(w_{i j}\right)$ exceeds a certain threshold. A bias value $\left(b_{1}\right)$ is also associated with activation threshold for each processing units as shown in Figure 2(a). Multiple layer perceptrons are used in a feed forward manner such that the signal flows from the input layer to the output layer as shown in Figure 2(b). During the training of the network, the weights are adjusted such that the error between the predicted and targeted output is minimized. For this purpose, an extensively popular back propagation algorithm is used which back propagates the error from the output layer to the input layer along with the adjustments in the weights.

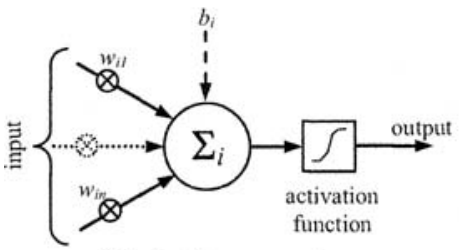

(a) single perceptron

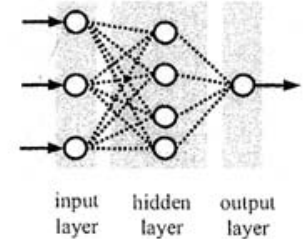

(b) multi-layer perceptron

Figure 2 - Perceptrons

\subsubsection{Architecture}

The architecture of the NN, i.e. the number of input-hidden-output layers, is intimately linked with the number of data used to train the network and plays an important role in predicting the output. Four different network architectures with a sigmoidal activation function have been developed in this work. The architectures is represented by the input-hidden-output nodes, with the inputs of $v, f, \alpha$, and $\bar{a}_{\text {thisp }}$,

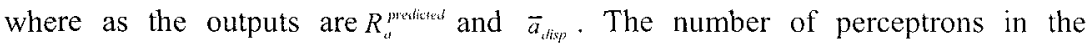


individual layers is selected based on the knowledge of the cutting process as well as the number of training data. For example, for the three input $(v, f, \alpha$, model, the output may be only $R_{a}^{\text {predicted }}$ with single hidden layer [3-4-1] and multiple hidden layer [3-2-2-1], but may also have both $R_{a}^{\text {predicted }}$ and $\bar{a}_{\text {disp }}$ as the outputs [3-3-2]. Again if $\bar{a}_{\text {disp }}$ is considered as an input to the surface generation (Jang, 1996) a [4-31] $\mathrm{NN}$ model is required.

\subsubsection{Training Functions}

The NN is trained, during which the weights corresponding to the connections get iteratively modified for minimizing the error between the predicted and target output values. The number of training data should be more than the total number of unknown parameters, i.e. the weights and the biases. A number of training functions have been used with the help of $M A T L A B^{T M}$ with different algorithms to update the weight and bias values.

\subsubsection{Implementation}

The 27 experimental data was separated into two sets of 21 and 6 for training and validation respectively such that the training set included the extreme values of the input parameters. Pre-processing of the input data was performed which included removal of the mean value and including unit deviation. A feed-forward multi layer perceptron network was used with different architectures and training functions. After initiating the weight matrix, the training of the network was carried on such that the mean square error was minimized and also repeated for consistent error values. Once the training is completed, the network was used to predict the output with the validation input data. The percentage prediction error $\left(\varepsilon_{N N}^{p}\right)$ given by the following equation is calculated for all the validation data, averaged and compared to select the most accurate network architecture as well as training functions.

$$
\varepsilon_{\text {int }}^{P}=\frac{\mid \text { Actual }- \text { Predicted } \mid}{\text { Actual }} \times 100
$$

\section{BIFURCATED OPTO-ELECTRICAL TRANSDUCER}

\subsection{Design and operating principle}

The bifurcated opto-electrical transducer shown in Figure 3, is a non-contact type displacement transducer, operating on the principle of scattering of laser beam, and had been used to measure tool wear (Choudhury, 1995) by monitoring the dimensional deviation during the turning operation. It is believed that the transducer can be used for monitoring on-line surface roughness which is superimposed on the dimensional profile, if tool wear, the primary cause of the dimensional deviation is minimized. The transducer consists of bundles of optical fibers with a diameter of $50 \mu \mathrm{m}$, a $10 \mathrm{~mW}$ continuous He-Ne Uniphase laser and SI 100 s photodiode. The single end section of the transducer is shown in Figure 3, where the inner bundles 
supply the laser beam which gets scattered from the rough surface and the reflected beams are sent to the photodiode through the outer annular bundles.

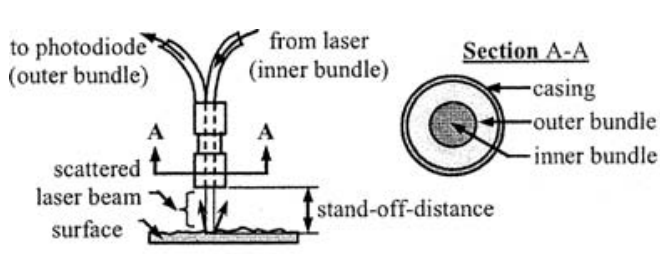

Figure 3-Bifurcated opto-electrical transducer

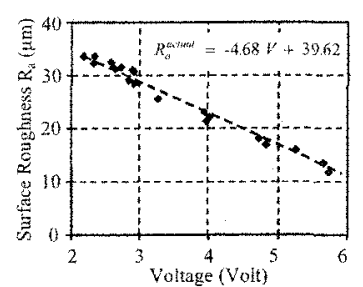

Figure 4 - Calibration chart

\subsection{Calibration}

Calibration of the bifurcated opto-electrical transducer is required to determine the output voltage $(V)$ in terms of the actual surface roughness $\left(R_{a}^{\text {actual }}\right)$ measured off-line. The calibration chart, shown in Figure 4, was obtained as a $V-R_{i a}$ function by measuring pre-machined surfaces with a Taylor-Hobson Surtronic digital surface profiler. The transducer was axially moved over the surface in the axial direction without cutting with a reference stand-off-distance (SoD) of $5.6 \mathrm{~mm}$. Measurements were taken at five different positions, and the equation of the best-fit line was calculated.

\section{EXPERIMENTAL SET-UP AND PROCEDURE}

The experimental set-up used for on-line measurement and monitoring of actual surface finish is shown in Figure 5. A cylindrical EN24 steel workpiece $(80 \mathrm{~mm}$ dia, $750 \mathrm{~mm}$ length) was divided into nine sections of equal lengths and was machined with a manual lathe (HMT LB25) using high-speed steel (10\% Cobalt) cutting tool. After turning each section with different feed, the tool was ground to minimize the effects of tool wear and rake angle. A Bruël Kjær 820361 accelerometer was mounted on the tool carriage to measure the accelerations of the tool-workpiece displacements.

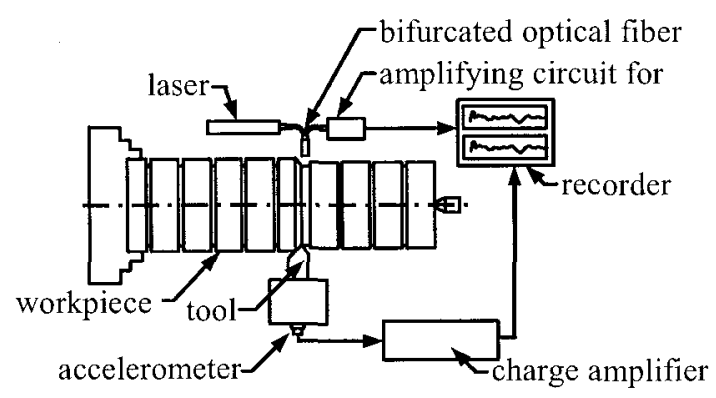

Figure 5-Schematic diagram of experimental set-up 
A $3 \mathrm{k}$ full factorial design experiment (Montgomery, 1997) was carried out for three inputs $(v, f, \alpha)$ with three levels (low, intermediate, high) having a total of 27 experiments. The ranges of the input factors $(v: 18.6-22.6 \mathrm{~m} / \mathrm{min} ; f: 0.50-$ $0.88 \mathrm{~mm} / \mathrm{rev} ; \alpha:-10^{\circ}-+10^{\circ}$ ) were selected from handbook (Oberg, 2000) and the machine capabilities such that $R_{c}^{\text {sctur }}$ was higher to facilitate the on-line measurement. The depth of cut was kept constant at $0.3 \mathrm{~mm}$, as it has the least effect on the surface roughness (Beauchamp, 1996).

\section{COMPARATIVE STUDY}

\subsection{Correlation between $R_{a}^{\text {predicted }}$ and $R_{a}^{\text {acturat }}$}

\subsubsection{Regression Models}

The minimum least square method incorporated within statistical package MINITAB ${ }^{\mathrm{TM}}$ was applied to calculate the coefficients $\left(\beta_{i}\right)$ of the models from 21 experimental data minimizing the prediction error $\left(\varepsilon_{r \mathrm{~g} g}^{p}\right)$ between the off-line measured $R_{\alpha}^{\text {cactual }}$ and calculated $R_{a}^{\text {preticteret }}$. The other 6 data corresponding to the intermediate levels of the inputs were used for calculating relative prediction error

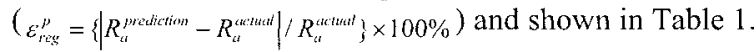

Table 1 -Comparison of $R_{a}^{\text {acruseit }}$ and $R_{u}^{\text {preatices }}$ for different regression models

\begin{tabular}{l|l|c}
\hline Model & $\varepsilon_{\text {reg }}^{p}(\%)$ \\
\hline \multirow{2}{*}{ Three Regressor } & Multiple Log-Linear & 20.71 \\
& Second Order Surface Response & 16.73 \\
\hline \multirow{2}{*}{ Four Regressor } & Multiple Log-Linear & 16.94 \\
& Second Order Surface Response & 13.82 \\
\hline
\end{tabular}

Analysis of Variance method and software $A N O V A^{T M}$ were used to compare the effect of each input parameter along with their levels on the output variable. Results shown that $v$ had the most significant effect on $R_{a}^{\text {preticesed }}$ followed by $\bar{a}_{\text {tiap }}, f$ and $\alpha$ and verified from literature (Escalona, 1998, Chen, 2001).

\subsubsection{Neural Network Models}

Similar to the regression analysis, the same 21 data were used to train the neural network and the 6 intermediate data to determine the prediction error ( $\varepsilon_{w *}^{p}$ ) between $R_{a}^{\text {cactitul }}$ and $R_{a}^{\text {prydictual }}$. All the above training functions were applied with a maximum number of training cycles of 10,000 to minimize the steady-state mean square error. The architecture, training function as well as $\varepsilon_{N Y}^{p}$ of the most accurate models with steady state error are given in Table2.

The above table shows that, NN is capable of predicting the actual surface roughness extremely accurately. As expected, more complex architectures or 
networks with higher number of hidden layers ([3-2-2-1]) give more accurate prediction, with the Levenberg-Marquardt training function working better for such architectures. It can also be observed that the prediction error for $\bar{a}_{\text {dis: }}$ is much higher as well as when the same is included as an input deteriorates the prediction capability for the actual surface roughness.

Table 2 - Comparison of $R_{a}^{\text {ortuat }}$ and $R_{a}^{\text {precticted }}$ for different NN models

\begin{tabular}{l|l|c}
\hline Architecture & \multicolumn{1}{|c|}{ Training Function } & $\varepsilon_{N N}^{\prime \prime}(\%)$ \\
\hline$[3-4-1]$ & Gradient Descent & 1.141 \\
{$[3-3-2]$} & Levenberg-Marquardt & 22.97 \\
{$[3-2-2-1]$} & Levenberg-Marquardt & 0.662 \\
{$[4-3-1]$} & Gradient Descent & 1.849 \\
\hline
\end{tabular}

5.2 Correlation between $R_{a}^{m-l i m e}$ and $R_{a}^{\text {actral }}$

The comparison between $R_{c}^{m-t m e}$ and $R_{i}^{\text {ectutal }}$ obtained from the 27 experiments is shown in Figure 6, which had a correlation of 0.891. If consider $R_{\mathrm{c}}^{\text {errmal }}$ as the

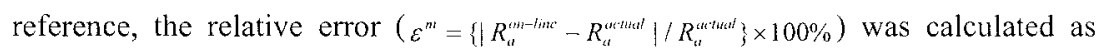
$14.4 \%$. A main source of this relative error may be relative tool-workpiece displacements and workpiece that change SoD and scattering conditions of the all laser beams from static during off-line measurements to dynamic during actual turning process.

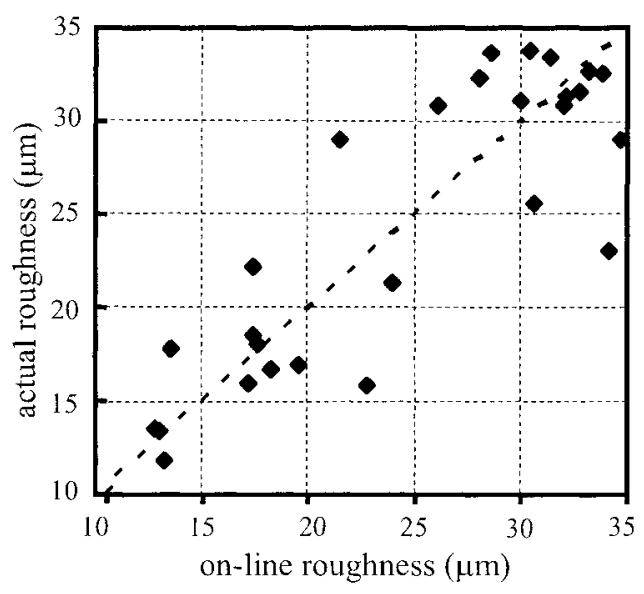

Figure 6 -Correlation between $R_{u}^{\text {gr-line }}$ and $R_{u t}^{\text {utrtut }}$

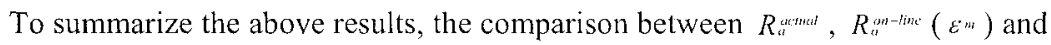
$R_{u}^{\text {predicted }}\left(\varepsilon_{r, g}^{p}, \varepsilon_{k N}^{p}\right)$ for the most accurate regression and $\mathrm{NN}$ model, with individual errors along with a $15 \%$ error $\left(\varepsilon_{15 \%}\right)$ range on $R_{a}^{\text {unturit }}$ is shown in Figure 7. 


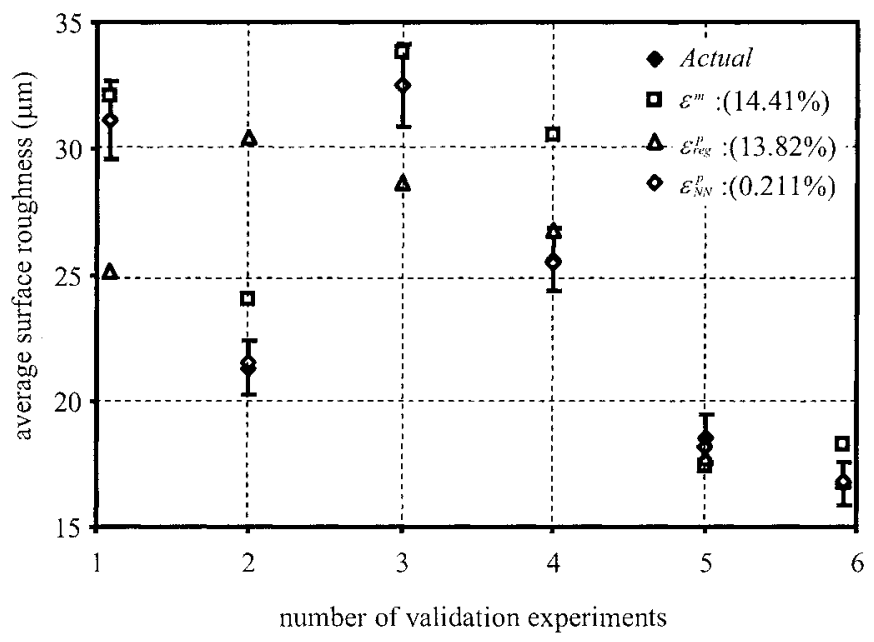

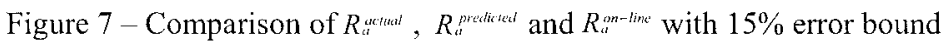

\section{CONCLUSIONS}

The comparative study of different mathematical models with respect to the actual average surface roughness and the predicted roughness showed that the prediction errors for regression models are higher than NN models for the same validation data, with a minimum error of $13.82 \%$ compared to $0.662 \%$ for $\mathrm{NN}$. However, the initiation of the weight matrix plays a vital role in the training of the $\mathrm{NN}$ and the subsequent selection of the optimally trained network needs much expertise.

The on-line surface roughness monitored using the developed bifurcated optoelectrical transducer closely follows the actual roughness with a correlation of 0.891 with a relative inaccuracy of $14.4 \%$ arising between them is primarily due to the relative tool-workpiece displacements and dynamic measurement conditions during the turning. Thus the transducer can be used for on-line monitoring of surface roughness in turning for practical applications within a tolerance of $15 \%$.

Results on comparison of the prediction errors of the modelling and the relative inaccuracy of the on-line monitoring indicate that transducer has better accuracy than most of the regression models. The ability of $\mathrm{NN}$ models to predict roughness beyond the input ranges will limit its application significantly. Moreover, the transducer has advanced potential for industrial applications where the entire surface profile is required rather than roughness measured at discrete locations. In such situations, the modeling would become substantially complicated for example for on-line control of the surface roughness in turning where identification of the cutting process dynamics is required. 


\section{ACKNOWLEDGEMENTS}

This study was partially supported by NSERC Discovery Grant R3440A01. The authors will also like to thank the technicians in the Manufacturing Science Lab, Indian Institute of Technology, Kanpur for their help to carry out the experiments.

\section{REFERENCES}

1. Abouelatta $O B$, Mádl J. Surface Roughness prediction based on cutting parameters and tool vibrations in turning operations. Journal of Material Processing Technology 2001; 118;269-77.

2. Beauchamp TM, Youssef AY, Masounave J. Effect of tool vibrations on surface roughness during lathe dry turning process. Computers and Industrial Engineering 1996;31(3-4);637-44.

3. Chen JC, Savage MD. Multiple regression-based multilevel in-process surface roughness recognition system in milling operations. The Journal of Technology Studies, Virginia Technical University $2001 ; 28-34$

4. Choudhury IA, Baradie MA El. Surface roughness prediction in turning of high strength steel by factorial design of experiments. Journal of Material Processing Technology 1997;67;55-61.

5. Choudhury SK, Ramesh S. On-line tool wear sensing and compensation in turning. Journal of Material Processing Technology 1995;49(3-4);247-54.

6. Dimla Jr DE, Lister PM, Leighton NJ. Neural network solution to the tool condition monitoring problem in metal cutting- a critical review of methods. International Journal of Machine Tool and Manufacture 1997;37(9);1219-41.

7. Escalona PM, Cassier Z. Influence of critical speed on the surface of finish of turned steel. Wear 1998;218;103-9.

8. Jang DY, Choi YG, Kim HG, Hasiao A. Study of correlation between surface roughness and cutting vibrations to develop an on-line surface roughness measuring technique in hard turning. International Journal of Machine Tool and Manufacture 1996;36 (4);453-63.

9. Lee CS, Kim SW, Yim DY. An in-process measurement technique using a laser for non-contact monitoring of surface roughness and form accuracy of ground surface. Annals of CIR.P1987;425-8.

10. Lin SC, Chang MF. A study on the effects of vibrations on surface finish using a surface topography simulation model for turning. International Journal of Machine Tool and Manufacture 1998;38(3);763-82.

11. Montgomery DC. Design and Analysis of Experiments. New York: John Wiley \& Sons, 1997.

12. Oberg E, Jones FD, Horton HL, Ryffell HH. Machinery's Hand Book, 26th Edition, Industrial Press, (2000).

13. Rangwala S, Dornfeld D. Sensor integration using neural networks for intelligent tool condition monitoring. Transaction of ASME: Journal of Engineering for Industry. 1981;112;219-28.

14. Risbood KA, Dixit US, Sahasrabudhe AD. Prediction of surface roughness and dimensional deviation by measuring cutting forces and vibrations in turning process. Journal of Material Processing Technology 2003; 132;203-14.

15. Sata T, Li M, Takata S, Hiraoka H, Li CQ, Xiao XG, Xing XZ. Analysis of surface ronghness in turning operation and its application. Annals of CIRP 1985;34(1);473-76.

16. Shriashi M. In-process measurement of surface roughness in turning by laser beams. Transaction of ASME: Journal of Engineering for Industry 1981;103;203-9 